\title{
Pricing of Coexisting Cellular and Community Networks
}

\author{
Patrick Maillé \\ IMT Atlantique / IRISA \\ Rennes, France \\ patrick.mailledimt.fr
}

\author{
Bruno Tuffin \\ Inria \\ Rennes, France \\ bruno.tuffineinria.fr
}

\author{
Joshua Peignier, Estelle Varloot \\ ENS Rennes \\ Rennes, France \\ first.lastdens-rennes.fr
}

\begin{abstract}
Community networks have emerged as an alternative to licensed-band systems (WiMAX, 4G, etc.), providing an access to the Internet with Wi-Fi technology while covering large areas. A community network is easy and cheap to deploy, as the network is using members' access points in order to cover the area. We study the competition between a community operator and a traditional operator (using a licensed-band system) through a game-theoretic model, while considering the mobility of each user in the area.
\end{abstract}

\section{INTRODUCTION}

Wireless technologies are becoming ubiquitous in Internet usage. Operators try to provide a whole wireless coverage on urban areas, in order to offer an Internet access to everyone, with a guaranteed quality. However, this system requires huge investment costs in terms of infrastructure and spectrum licenses. This has repercussions on the subscription fees, which can be large enough for users to prefer other options. Because of this, community networks have been imagined as an alternative. The principle is simple: when a user subscribes to a community network, he sets an access point where he lives (and is responsible for its maintenance), which can be used by all members of the community network. As a counterpart, he gains access to the Internet through every access point belonging to the community network. This approach presents the advantage that the infrastructure is cheaper and easier to maintain, from a provider perspective. However, the quality of service cannot be guaranteed, since it depends on the size of the community. Currently, the largest community operator is $\mathrm{FON}^{1}$.

From the user point of view, a community network has the particularity of having both positive and negative externalities, i.e., having more subscribers is both beneficial (larger coverage when roaming) and a nuisance (more traffic to serve from one's access point). An analysis of those effects and on the impact of prices, with users being heterogeneous in terms of their propension to roam, is carried out in [1]. In the present paper, we add another dimension, that is, how-i.e., whereusers roam. Also, we consider that users can choose between two competing providers, a "classical" one and one operating a community network, that compete over prices.

Community networks have already been studied under a game-theoretic framework, with operators as players. In [2],

\footnotetext{
${ }^{1}$ https://fon.com/
}

the authors first study how a community network evolves, depending on its initial price and coverage, and then investigate using a game-theoretic framework [3] the repartition of users having the choice between a community network and an operator on a licensed band. The competition is first studied when each player decides its price once and the size of the community network changes over time. Then a discrete-time dynamic model is studied, where operators can change their price at each time step, taking into account the preferences of the users concerning price and coverage. The authors show the existence of one or several Nash equilibria under specific conditions. An extension in [4] investigates whether it is profitable for a licensed-band operator to complement the service it provides with a community network service. It is shown that this is generally not the case, as users will more likely choose the (less profitable) community network. In [5], the same authors study an optimal pricing strategy for a community network operator alone in both static and semidynamic models, while considering a mobility factor for each user (e.g., each user makes requests, but not all in the same spot). They also allow the operator to set different prices for each user. In the following, we will refer to the traditional operator as the classical ISP (Internet Service Provider).

In this article, we study a model similar to both [2] and [5]. In [2] the users all present the same characteristics while in [5] there is a mobility factor but the paper considers a community network alone. We consider here a more general and realistic framework: users are considered located in places heterogeneous in terms of attractiveness for connections (an urban area is more likely to see connections than the countryside). Moreover, their mobility behavior is also heterogeneous: they do not all plan to access the Internet from the same places. Instead of a mobility parameter, we rather consider a density function, which represents the probability that a user makes a request while being near the access point of another user. But in our paper, all users will have the same sensitivity toward quality; indeed, our goal is rather to focus on the impact of geographical locations of spots and connections on users' subscription and on the competition between the operator and the community network. The model is analyzed using noncooperative game theory [3], [6]. The decisions are taken at different time scales: first the networks fix their price, and then users choose which network to subscribe to. We illustrate 
on different scenarios that for fixed subscription prices to the ISP and the community network, several equilibria on the repartition of users can exist; the one we can expect depends on the initial mass of the subscribers to the community network. The pricing competition between operators is played anticipating the choice of users.

The paper is organized as follows. Section II presents the model; the basic notions are taken from the literature, but we extend it with the modeling of mobility via a continuous distribution. In Section III, we describe how, for fixed subscription prices, the repartition of users is determined. In Section IV, we introduce the pricing game between the operator and the community network, as well as our method to compute a Nash equilibrium. Section V presents two scenarios as examples of application of our method.

\section{MODEL DEFINITION}

We present here the basic elements of the model taken from the literature, mainly [2], which we complement with more heterogeneity among users related to location and mobility, as well as the possible nuisance from providing service to other community members.

\section{A. Actors and strategies}

To study the competition between a community operator and a classical ISP, we need to define a model for the profit of each operator, but also for users, in order to explain what will make them choose a service rather than the other one. The decisions of these actors are taken at different time scales, defining a multilevel game:

1) First, the classical ISP and the community network play non-cooperatively on the subscription prices, in order to maximize their own revenue (expressed as the product of price and mass of users).

2) Given the prices and qualities of service, users choose their network based on price (we assume a flat-rate pricing is applied by each operator) and quality of service. We will describe how, depending on an initial repartition between operators, users can switch operators up to a situation when nobody has an interest to move.

The results of the game for the operators are given once all users have settled on an operator (if any). Even though the operators play first (subscription is impossible until the price of subscription is set), they are assumed to make their decision strategically, anticipating the subsequent decisions of users. Hence the game is analyzed by backward induction [3]: we determine the user choices for any fixed prices, and consider that operators are able to compute those choices when selecting their prices.

\section{B. Modeling of users, quality, and mobility}

We consider a continuum of users characterized by their type $u$; this type typically represents a home location. In the following, we will not distinguish between a location $u$ and the user $u$ living there. Let $\Omega$ be the space of users and $f$ their density over space $\Omega$ (with $\int_{\Omega} f(v) \mathrm{d} v=1$ ). We also assume that $\Omega$ is the support of $f$, i.e., $f(u)>0$ for all $u \in \Omega$.

Let $D$ be the subset of $\Omega$ of users subscribing to the community network; we call it the domain, since it also represents the domain of coverage of the community network.

Each user makes requests when using the Internet. Let $m(v)$ be the average number of communication requests of user $v$ per time unit.

Depending on their location, users may also present different mobility patterns. To express this heterogeneity, define for each user $u$, the density function $g(v \mid u)$ that a request from $u$ occurs at $v$. Note that users may move to uninhabited regions: we aggregate those regions into one item, denoted by $\perp$, and we define the set of mobility locations as $\bar{\Omega}:=\Omega \cup \perp$. Then, over a location area $A \subset \bar{\Omega}$, the probability that a type- $u$ user's request is in $A$ (rather than $\bar{\Omega} \backslash A$ ) is $\int_{A} g(v \mid u) \mathrm{d} v$.

If we define $n(u)$ as the density (number per space unit) of requests at $u$ from users of the community network, it can be computed as

$$
n(u)=\int_{D} g(u \mid v) m(v) f(v) \mathrm{d} v .
$$

The quality of a given service is defined as the probability that a request is fulfilled. For the ISP assumed to have a full coverage, it is therefore 1 , in line with the literature. For a user $u$, the quality of the community network will depend on whether the requests by $u$ are generated when in the coverage domain, hence it can be computed as

$$
q_{u}=\int_{D} g(v \mid u) d v
$$

\section{User preferences}

How will User $u$ decide whether to subscribe to the classical ISP, to the community network, or to none of them? Following [2], [4], define $U_{I}(u), U_{C}(u)$ and $U_{\emptyset}(u)$ as the respective utility functions for choosing the classical ISP, the community network, or none. These functions depend on the price the user has to pay, the quality of the service he is provided, and his sensitivity toward quality. As in [2], [4], we consider a simple quasi-linear form for utilities: a user $u$, whose sensitivity toward quality is denoted by $a$ and who benefits from service quality $\tilde{q}$ (assumed in the interval $[0,1]$ ) at price $p$ perceives a utility $a_{u} \tilde{q}-p$. Note that in [2], [4] the sensitivity parameter $a$ depends on the user type, $u$, but we limit ourselves to a constant value $a$ for all users since our goal is to focus on the geographical heterogeneity of users.

In addition, as in [1] we consider a disturbance factor for the community network: satisfying requests for other members can indeed become an annoyance, which we model through a negative term $-c n(u)$ in the utility function, with $c$ a unit cost per request at $u$. Here we assume that the nuisance is due to WiFi spectrum usage, hence it depends on the total density of requests in $u$ and is independent of the density of users at $u$.

Let $p_{I}$ and $p_{C}$ be the respective flat-rate subscription fees to the ISP and to the community network, respectively. 
We assume users are rational: a type- $u$ user will choose the network providing the largest utility (or no network), where the utilities at the community network, the ISP or for not subscribing to any are respectively (recall those functions depend on the set $D$ of users in the community network)

$$
\begin{aligned}
U_{C}(u) & =a q_{u}-p_{C}-c n(u) \\
U_{I}(u) & =a-p_{I} \\
U_{\emptyset}(u) & =0,
\end{aligned}
$$

where $U_{\emptyset}(u)$ is used to say that users with negative utilities at the operators do not subscribe to any of them; we also assume that users with null utilities at the operators do not subscribe.

In the following, we will therefore always assume that $p_{I}<a$, because otherwise, the classical operator would get no subscriber (as for all $u$, we would have $U_{I}(u) \leq 0$ and all users prefer the no-subscription option over the classical operator). With the same argument, we assume that $p_{C}<a$. We now have, for all $u, U_{I}(u)>0$, which implies that each user will necessarily subscribe to one operator, since they strictly prefer the classical operator over the no-subscription option.

However, the repartition between the classical ISP and the community network is not trivial, since the utilities expressed above, which determine user choices, also depend on user choices through the set $D$. Hence the notion of equilibrium (or fixed-point), which we define and analyze in Section III.

\section{Operators' model}

The utilities for the classical ISP and the community operator are simply defined as their profits. For each operator, the profit depends on the price it chose, and on the number of users subscribing to its service, which depends on both prices.

Let $d_{I}$ and $d_{C}$ be the number (or mass) of users subscribing respectively to the classical ISP and to the community operator. For a set $D \subset \Omega$ of users subscribing to the community network (which depends on prices as we see later on), those masses can be written as

$$
\begin{aligned}
d_{I} & =\int_{\Omega \backslash D} f(v) d v \\
d_{C} & =\int_{D} f(v) d v .
\end{aligned}
$$

The utilities are then expressed by

$$
\begin{aligned}
V_{C} & =d_{C} p_{C} \\
V_{I} & =d_{I} p_{I}-\chi_{I},
\end{aligned}
$$

where $\chi_{I}$ is the infrastructure cost for the ISP. Each operator chooses (plays with) its price to maximize its revenue, but that revenue also depends on the decision of the competing operator which can attract some customers, hence the use of non-cooperative game theory to solve the problem.

\section{USER EQUILIBRIUM}

With the characterization of user behavior above, we aim in this section at determining if, for fixed subscription prices, there is an equilibrium user repartition among operators, and also if it is unique. We first define what such an equilibrium is. We consider here that prices $p_{I}$ and $p_{C}$ have already been decided.

\section{A. Definition and characterization}

Definition 1. A user equilibrium domain is a domain $D \subset \Omega$ such that no user, in $D$ or in $\Omega \backslash D$ has an interest to change his choice of network. Mathematically, this means that

$$
\begin{aligned}
\forall u \in D & U_{C}(u) \geq U_{I}(u) \\
\forall u \in \Omega \backslash D & U_{C}(u) \leq U_{I}(u) .
\end{aligned}
$$

Consider a User $u$. For a given domain $D$, he will prefer the community network if $U_{C}(u) \geq U_{I}(u)$, that is, if $a\left(q_{u}-\right.$ 1) $+\left(p_{I}-p_{C}\right)-c n(u) \geq 0$.

Let us define the domain-dependent function $\Phi_{D}: \Omega \mapsto \mathbb{R}$ as the difference $U_{C}(u)-U_{I}(u)$, that is,

$\Phi_{D}(u):=a\left(\int_{D} g(v \mid u) d v-1\right)+\left(p_{I}-p_{C}\right)-c \int_{D} g(u \mid v) m(v) f(v) \mathrm{d} v$.

Then $D$ is a user equilibrium domain if and only if $\begin{cases}\Phi_{D}(u) \geq 0 & \forall u \in D \\ \Phi_{D}(u) \leq 0 & \forall u \in \Omega \backslash D .\end{cases}$

Example 1. Consider the case of users with homogeneous mobility behavior, that is, where $g(v \mid u)$ does not depend on $u$ so we only denote it by $g(v)$. From (1), we also get that the quality $q_{u}$ does not depend on $u$ : all the community network users experience the same quality, which we denote by $q$ and equals $\left.\int_{D} g(v) d v\right)$. Moreover $n(u)=g(u) \int_{D} m(v) f(v) d v=$ $M g(u)$ with $M:=\int_{D} m(v) f(v) d v$ the total request mass from community network users. At a user equilibrium $D$, User $u$ prefers the community network if and only if

$$
a(q-1)+\left(p_{I}-p_{C}\right)-c M g(u) \geq 0 .
$$

The domain $D$ is then made of all users $u$ with attractiveness $g(u)$ below a threshold.

\section{B. Existence and uniqueness}

Proposition 1. A user equilibrium is not unique in general.

Proof. An example of non uniqueness is shown in Section V-A when users present a homogeneous mobility pattern $g(v \mid u)=$ $g(v) \forall u$.

Proposition 2. $D=\emptyset$, that is no user subscribes to the community network, is a user equilibrium (but not necessarily the only one) if and only if $p_{I} \leq p_{C}+a$. In words, if the difference of price between the community network and the ISP is not large enough (it has to be larger than a), no user subscribing to the community network is a user equilibrium, even if not necessarily the unique possibility.

Proof. $D=\emptyset$ is a user equilibrium if and only if, when there are no community network users, $U_{C}(u) \leq U_{I}(u) \forall u \in \Omega$; that is, $-a+\left(p_{I}-p_{C}\right) \leq 0$, i.e., $p_{I} \leq p_{C}+a$.

We can also consider the other case of "degenerate" equilibrium, that is, when all users in $\Omega$ subscribe to the community network. 
Proposition 3. $D=\Omega$, is a user equilibrium if and only if $\Phi_{\Omega}(u) \geq 0$ for all $u \in \Omega$.

Corollary 1. Under our assumption $p_{I}<a$, there always exists at least one user equilibrium.

Proof. Since we have assumed $p_{I}<a$, Proposition 2 holds.

\section{A dynamic view}

Given that several user equilibria might exist, which one would be observed in practice? This may depend on a dynamic evolution of subscriptions: We can study how users make their choice, and how the repartition evolves, depending on an initial situation.

If a user $u$ is associated to the ISP (resp. community network) but $U_{C}(u)>U_{I}(u)$ (resp. $U_{C}(u)<U_{I}(u)$ ) then it will switch to the other operator. Without loss of generality, we can first partition users by assuming that those with the largest $U_{C}(u)-U_{I}(u)$ subscribe to the community network and the others to the ISP (a natural move to that situation will occur otherwise). We can relate this to the function $\Phi_{D}(x)$ defined in (2). For a given $D$, users $u \notin D$ (resp. $u \in D$ ) with the largest value $\Phi_{D}(u)>0$ (resp. lowest value $\Phi_{D}(u)<0$ will have an incentive to switch operator and join (resp. leave) $D$. Hence, $D$ will change up to a moment when no user has an interest to move, that, up to reaching a user equilibrium as defined above.

All this will be made more specific and clearer in Section V on the analysis of two scenarios.

Depending on the initial situation (that is, the initial mass of users subscribing to the community network), we may end up in different user equilibria. We can assume that the community network will offer free subscriptions, or make offers to users so that an initial point will allow to lead to different equilibria.

\section{Stability}

Among user equilibrium domains, some are more likely to be observed. They are the so-called stable user equilibrium domains, which can basically be defined as domains that are stable to small perturbations in the following sense.

Definition 2. A user equilibrium domain $D$ is said to be stable if there exists $\varepsilon>0$ such that $\forall D^{\prime}$ with $\int_{\left(D \cup D^{\prime}\right) \backslash\left(D \cap D^{\prime}\right)} f(v) \mathrm{d} v \leq \varepsilon$ (that is, any $D^{\prime}$ with "measure" close enough to $D$ ), then starting from $D^{\prime}$ the user repartition will converge to $D$.

The following straightforward result establishes that there always exists at least one stable equilibrium.

Proposition 4. If for all $u \in \Omega$, the ratio of the densities $g(\cdot \mid u) / f(\cdot)$ is upper-bounded on $\Omega$, then for any price profile $\left(p_{I}, p_{C}\right)$ with $p_{I}<a$, the situation $D=\emptyset$ is a stable equilibrium. Similarly, the other degenerate equilibrium $D=\Omega$ is stable if $\Phi_{\Omega}(u)>0$ for all $u \in \Omega$.
Proof. From Corollary $1, D=\emptyset$ is a user equilibrium domain. Since $p_{I}<a$, (2) yields $\Phi_{D}(u)=p_{I}-a-p_{C}<0 \forall u \in \Omega$. We also have from (2) that for any domain $D^{\prime}$ and any $u$,

$$
\Phi_{D^{\prime}}(u) \leq p_{I}-a-p_{C}+a \int_{D^{\prime}} g(v \mid u) \mathrm{d} v .
$$

But when the ratio $g(\cdot \mid u) / f(\cdot)$ is upper-bounded by some value $L$, then the integral $a \int_{D^{\prime}} g(v \mid u) \mathrm{d} v$ is smaller than $a L \int_{D^{\prime}} f(v) \mathrm{d} v$. Therefore, with $\varepsilon<\frac{p_{C}+a-P_{I}}{a L}$, for a domain $D^{\prime}$ such that $\int_{D^{\prime}} f(v) \mathrm{d} v \leq \varepsilon$ all users in $D^{\prime}$ would be better off switching back to the ISP, hence $D=\emptyset$ is a stable equilibrium domain.

Further characterizations are provided in Section $\mathrm{V}$ for specific scenarios.

\section{Pricing Game}

For any price pair $\left(p_{I}, p_{C}\right)$, being able to characterize all stable user equilibria, we can reasonably assume that the community network will set up things (again, by initial offers/bargains) such that the largest (defined in terms of demand $d_{C}$ ) stable user equilibrium domain is reached in the end. Therefore, for given prices, we will be able to compute the corresponding values of the utility functions $V_{C}$ and $V_{I}$ of each operator. Hence providers can non-cooperatively play the pricing game where the community network chooses $p_{C}$ and the ISP chooses $p_{I}$, each operator trying to maximize its utility function. The solution concept is the classical Nash equilibrium [3], a pair $\left(p_{I}^{*}, p_{C}^{*}\right)$ from which no provider can improve its revenue from a unilateral price change.

\section{ANALYSis AND DisCUSSION OF TWO SCENARIOS}

\section{A. Users with a homogeneous mobility pattern}

We first consider the simplest situation where the mobility pattern is the same for all users, which means that $g(v \mid u)$ does not depend on $u$, that is $g(v \mid u)=g(v) \forall u$ as treated in Example 1. From this assumption, $q_{u}=q$ does not depend on $u$ (but still depends on $D$ ). We also get a much simpler expression for $\Phi_{D}$, which is now: $\forall u \in \Omega$,

$$
\Phi_{D}(u)=a(q-1)+\left(p_{I}-p_{C}\right)-c M g(u),
$$

which depends on $u$ only through the term $g(u)$, with $q=\int_{D} g(v) \mathrm{d} v$ and $M=\int_{D} m(v) f(v) \mathrm{d} v$. From such an expression, we can show that with an homogeneous mobility pattern, user equilibria have a specific form.

1) Characterization of user equilibria:

Proposition 5. Assume that location attractiveness values are distributed regularly over $\Omega$ : i.e. mathematically, that for all $y \in \mathbb{R}^{+}$, the mass of users with the specific value $g(u)=y$ is null. Then a non-degenerate user equilibrium domain has the form $D_{x}:=\{u \in \Omega \mid g(u) \leq x\}$ for a given $x \geq 0$, with $x$ solution of

$\underbrace{a\left(\int_{D_{x}} g(v) \mathrm{d} v-1\right)+p_{I}-p_{C}-c x \int_{D_{x}} m(v) f(v) \mathrm{d} v}_{:=\Psi(x)}=0$. 
In the above characterization, $x$ is a threshold such that all users $u$ with mobility attractiveness density $g(u)$ below $x$ subscribe to the community network .Remark that $\Psi(x)$ corresponds to $\Phi_{D_{x}}(u)$ for a user $u$ such that $g(u)=x$; and since $D_{x}$ is continuous in $x$ under our assumption, $\Psi$ is also a continuous function of $x$.

Proof. See Appendix A.

We can characterize, among all domains $D_{x}$, which ones will actually be user equilibrium domains, and the corresponding dynamics.

Assume that the set of subscriber to the community network is of the form $D_{x}=\{u \in \Omega: g(u) \leq x\}$ for some $x \in \mathbb{R}^{+}$.

- If $\Psi(x)>0$ and $D_{x} \neq \Omega$, it means that users $u$ with $g(u)$ just above $x$ are associated with the ISP and are those with the largest utility difference and incentive to switch to the community network (indeed, from (3) that utility difference $\Phi_{D_{x}}(u)$ is continuous and strictly decreasing in $g(u)$ ); hence they switch such that $x$ and $D_{x}$ increase;

- If $\Psi(x)<0$ and $D_{x} \neq \emptyset$, it is the opposite situation: users $u$ with value $g(u)$ just below $x$ are with the community network but have the largest incentive to switch to the ISP; hence $x$ and $D_{x}$ decrease;

- If $\Psi(x)=0$, all users $u \in \Omega$ are such that $\Phi_{D_{x}}(u) \geq$ 0 , hence have no interest to switch; we are then in an equilibrium situation.

We thus end up with the following characterization of user equilibrium domains. Let $y:=\sup _{u \in \Omega}\{g(u)\}$ (possibly $\infty$ ), such that $D_{y}=\Omega$.

- If $\Psi(y) \geq 0$ then $D=\Omega$ (all users subscribe to the community network) is an equilibrium;

- Since $\Psi(0)=-a+p_{I}-p_{C} \leq 0$ by assumption, $\emptyset$ is always a user equilibrium domain (no users associated with the community network);

- If $\Psi(x)=0, D_{x}$ is a user equilibrium domain.

2) Stable equilibria: Among all user equilibrium domains, we can characterize the stable ones.

Proposition 6. As suggested by the dynamics described in Subsection III-C, we consider that the community network subscriber set $D$ is always of the form $D_{y}$ for some $y$. Then if $\Psi(x)=0$ and $\Psi^{\prime}(x)<0, D_{x}$ is a stable equilibrium.

Proof. Assume a small variation, from $x$ to $x^{\prime}=x \pm \varepsilon$, in $D$ (hence, from $D_{x}$ to $D_{x^{\prime}}$ ). If $\Psi^{\prime}(x)<0$, for $\varepsilon$ small enough, $\Psi\left(x^{\prime}\right)>0$ (resp. $<0$ ) if $x^{\prime}<x$ (resp. $x^{\prime}>x$ ); hence users $u$ with $g(u)$ between $x^{\prime}$ and $x$ are incentivized to switch back to their initial choice, driving back to the (then stable) equilibrium domain $D_{x}$.

3) Nash equilibria for the pricing game between operators: For any pair $\left(p_{C}, p_{I}\right)$, we consider that the largest equilibrium domain is selected. Operators then play a non-cooperative game to determine their optimal strategy [3], [6]. The output concept is that of a Nash equilibrium, a point $\left(p_{C}^{*}, p_{I}^{*}\right)$ such that no operator has an interest to unilaterally move from, because it would decrease its utility (revenue). Because of

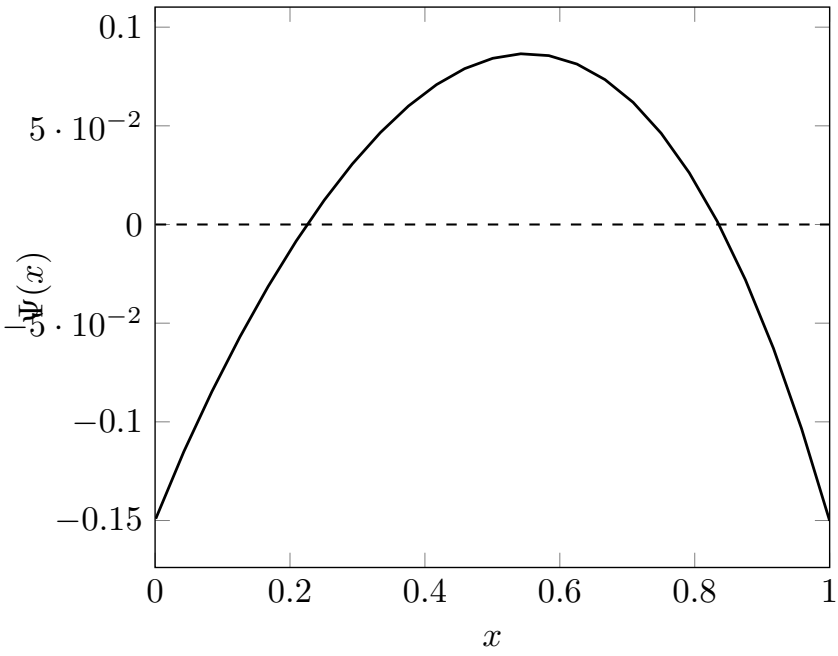

Fig. 1. $\Psi(x)$ when $\lambda=1.0$.

analytical intractability, we are going to study the existence, and characterize, Nash equilibria numerically, for specific parameters values; the procedure can be repeated for any other set of parameters.

4) Examples: We first show situations where there are several user equilibria, and even several stable user equilibria. We then discuss the solution of the pricing game between operators.

Example 2. Consider $\bar{\Omega}=\Omega=\mathbb{R}^{+}$, i.e., users are placed over the positive line (negative values could be described as the sea). We assume:

- $m(u)=1$, i.e., all users generate the same amount of requests;

- $f(u)=\alpha /(1+u)^{1+\alpha}$ with $\alpha>0$. In other words, users are located according to a Pareto distribution, potentially with infinite expected value. The closer to the 0 value (which can be thought of as the town center), the more users you can find.

- $g(u)=\lambda e^{-\lambda u}$, meaning that connections are exponentially distributed with rate $\lambda$, with more connections close to 0 ; even far-away users are more likely to require connections there.

With these functions, noting that $g$ is strictly decreasing, the set $D_{x}$ is simply the interval $[\ln (\lambda / x) / \lambda,+\infty)$ when $x \in(0, \lambda]$, $D_{x}=\Omega$ when $x>\lambda$, and $D_{x}=\emptyset$ when $x=0$. It gives

$$
\Psi(x)=-a(1-x / \lambda)+p_{I}-p_{C}-c x\left(\frac{1}{1+\ln (\lambda / x) / \lambda}\right)^{\alpha}
$$

for $x \in[0, \lambda], \Psi(x)=p_{I}-p_{C}-c x$ for $x>\lambda$, and $\Psi(0)=$ $p_{I}-a-p_{C}$. Note that the assumption made in Proposition 5 holds here, hence $\Psi$ is continuous over $R^{+}$.

Three outcomes are illustrated in the next three cases for $\lambda$ when $\alpha=1.2, a=1, c=1, p_{I}=0.95$ and $p_{C}=0.1$. In Figure 1, there are two solutions for $\Psi(x)=0$, with only the second one leading to a stable equilibrium domain (in addition to $D_{0}=\emptyset$, which is stable too from Proposition 4 , for which 


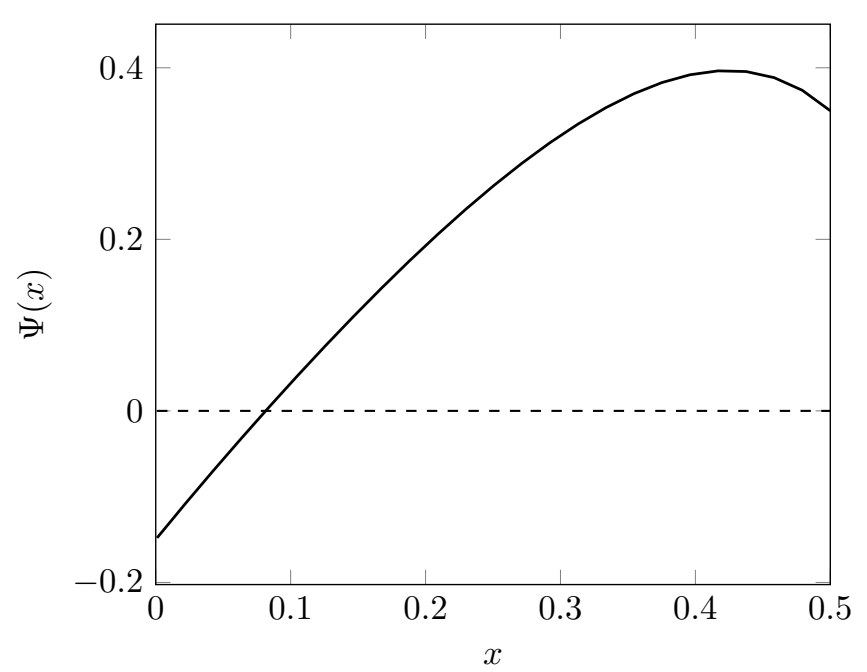

Fig. 2. $\Psi(x)$ when $\lambda=0.5$.

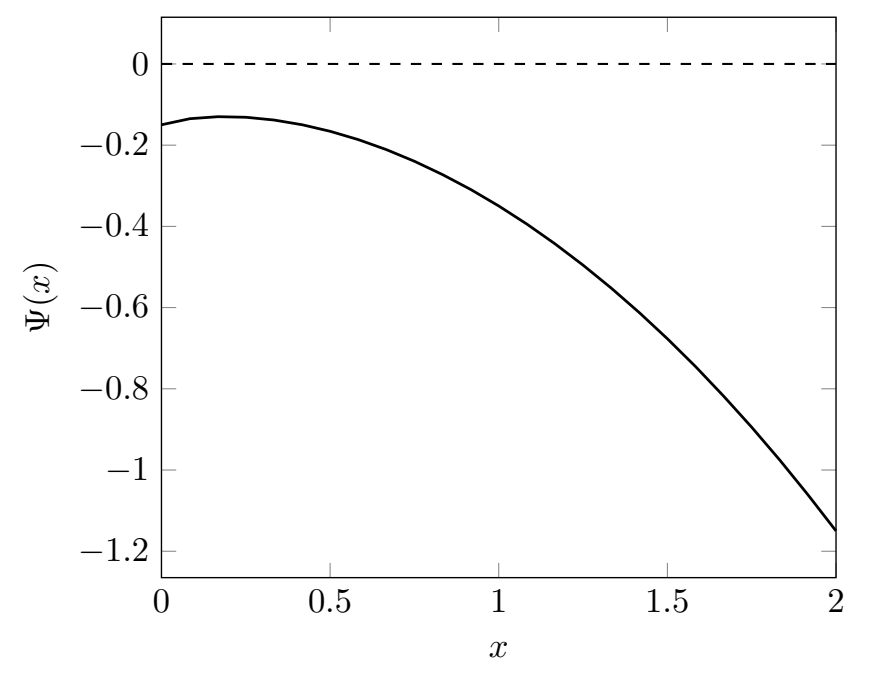

Fig. 3. $\Psi(x)$ when $\lambda=2$.

the assumptions also hold). In Figure 2, $\Psi(x)=0$ has only one (unstable) solution but $D_{0}=\emptyset$ and $D_{0.5}=\Omega$ (because $\Psi(0.5)>0)$ are stable equilibrium domains. In Figure 3 there is no solution to $\Psi(x)=0$, and $\emptyset$ is the only equilibrium domain. Remark that $\Omega$ is a (stable) equilibrium domain if $\Psi(\lambda)=p_{I}-p_{C}-c \lambda \geq 0 .^{2}$

Assuming now that the community network plays such that the largest equilibrium domain is selected (thanks to discounts for example), we can can draw the best responses of the pricing game between operators. It is for example displayed in Figure 4 for specific parameter values, here when the infrastructure cost for the ISP is $\chi_{I}=0$. With these parameters, the community network is able to get a positive demand only if $p_{I} \geq 0.76$. $d_{C}$ is then jumping from 0 to 0.574 and is readily and slightly increasing to 0.678 when $p_{I}=0.99$. We actually have here a price war where each operator has

\footnotetext{
${ }^{2}$ Note that with other functions, depending on the variations of $f$ and $g$, an arbitrary number of solutions of $\Psi(x)=0$ can be obtained.
}

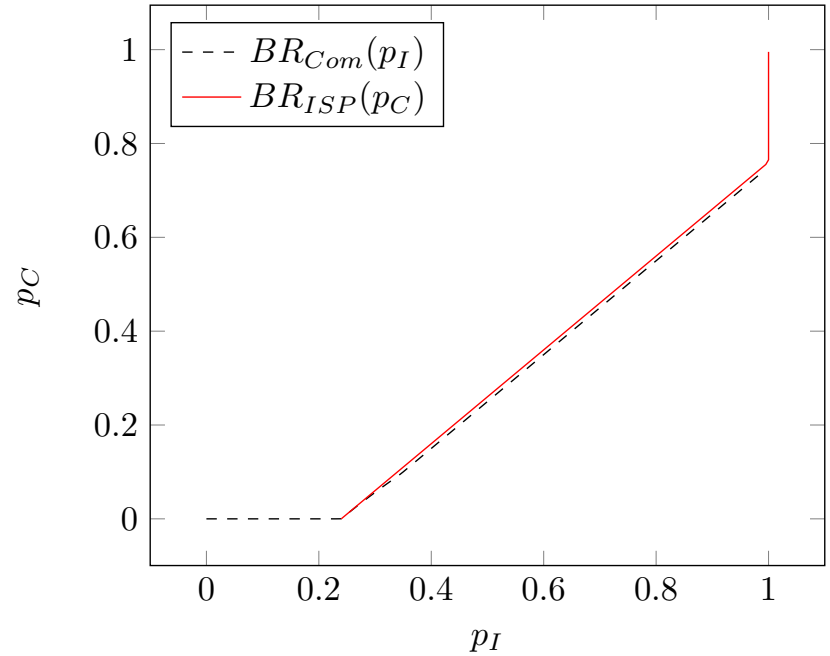

Fig. 4. Best responses in the pricing game when $\lambda=0.25, \alpha=1.2, a=1$, $c=1$, and $\chi_{I}=0$

an interest to give a price just below that of the opponent, and we end up with a Nash equilibrium $\left(p_{I}=0.23985, p_{C}=0\right)$ where one operator stops with the zero price. Due to price war, only one operator survives. With an infrastructure cost $\chi_{I}=0$, it is the ISP, but we note that the threat of the development of a community network significantly decreases the price set by the ISP (whose monopoly price was 1 as can be seen on Figure 4 when $p_{C}$ is prohibitively high). Also, there is a threshold on $\chi_{I}$ over which it is the community network that survives. Indeed, remark that the value of $\chi_{I}$ does not change the best-response values since it appears as a constant in the expression of $V_{I}$, but the game stops as soon as one of the two providers gets a zero revenue. The revenue of the ISP will go to zero before that of the community network if $\chi_{I}>0.23$.

\section{B. Several populations}

We slightly modify the model such that $\Omega=\mathbb{R}^{+}$with a mass of users in 0 , seen as a town (probability/mass $\pi_{0}$ ) while others with $u>0$ are regularly distributed over the "countryside" with conditional density $f$. In terms of mobility, we assume that users at $u=0$ do not move, while those at $u>0$ have a probability $\pi_{1}$ to call from 0 and a conditional density (when connecting from another place) $g(v)$ to make a connection from $v>0$. With those assumptions, remark that $q_{0}=1$ (resp. $q_{0}=0$ ) if the community network is chosen (resp., not chosen) at 0 , and for $u>0$, $q_{u}=\pi_{1} \mathbb{1}_{\{0 \in D\}}+\left(1-\pi_{1}\right) \int_{D^{1}} g(v) d v$ where $D^{1}=D \cap(0, \infty)$ is $D$ excluding 0 and $\mathbb{1}_{\{\cdot\}}$ is 1 if the condition is satisfied and 0 otherwise.

The number of connections to a member of the community network at 0 (assuming $0 \in D$ ) is then $n(0)=$ $\pi_{0} m(0)+\left(1-\pi_{0}\right) \pi_{1} \int_{D^{1}} m(v) f(v) d v$ and $n(u)=g(u)(1-$ $\left.\pi_{0}\right) \int_{D^{1}} m(v) f(v) d v$ for $u>0$. The level of annoyance (interferences, etc.) is again assumed linear in $n(\cdot)$, leading 
to

$$
\begin{aligned}
\Phi_{D}(0)=( & \left.p_{I}-p_{C}\right) \\
& -c\left(\pi_{0} m(0)+\left(1-\pi_{0}\right) \pi_{1} \int_{D^{1}} m(v) f(v) d v\right) \\
\Phi_{D}(u)=a & \left(\pi_{1} \mathbb{1}_{\{0 \in D\}}+\left(1-\pi_{1}\right) \int_{D^{1}} g(v) d v-1\right) \\
& +\left(p_{I}-p_{C}\right)-c\left(g(u)\left(1-\pi_{0}\right) \int_{D^{1}} m(v) f(v) d v\right) .
\end{aligned}
$$

Assuming that $0 \in D$ or not, the above last equation tells us as in the previous subsection that $D^{1}$ is of the form $D_{x}^{1}=$ $\{u: g(u) \leq x\}$ for some value $x$. Exactly as in the previous homogeneous case, there might be several solutions, and we will assume that the selected one will be a stable one leading to the largest market share (revenue) for the community network.

The more subscribers of the community network, the less likely users in 0 will subscribe because since they do not move, they experience only losses from an increased number of subscribers. But there might be a risk of oscillations on the user equilibrium. Indeed, if $x$ is small enough, then users in 0 subscribe. This increases the interest for others to subscribe, leading to a larger value of $x$, which might deter users in 0 , and so on.

Example 3. We again consider $m(u)=1$,

$f(u)=\alpha /(1+u)^{1+\alpha}$ with $\alpha>0$, and $g(u)=\lambda e^{-\lambda u}$. Then users in 0 join iff for $D^{1}=D_{x}^{1}=[\ln (\lambda / x) / \lambda,+\infty)$, they prefer the community network over the ISP, knowing that there is coverage in 0, i.e., iff

$$
\underbrace{\left(p_{I}-p_{C}\right)-c\left(\pi_{0}+\left(1-\pi_{0}\right) \pi_{1}\left(\frac{1}{1+\ln (\lambda / x) / \lambda}\right)^{\alpha}\right)}_{:=\Phi_{D}(0)} \geq 0,
$$

or

$$
x \leq \lambda e^{\lambda\left(1-\left(\frac{\left(1-\pi_{0}\right) \pi_{1}}{\left(p_{I}-p_{C}\right) / c-\pi_{0}}\right)\right)^{1 / \alpha}} .
$$

Note that $\Phi_{D}$ is a slight abuse of notation, since it corresponds to $D=\{0\} \cup D_{x}^{1}$ or just $D_{x}^{1}$ whether users at 0 join.

To get a non-degenerate equilibrium, $x \in[0, \lambda]$ must be a solution of

$$
\begin{aligned}
\Psi(x)= & a\left(\pi_{1} \mathbb{1}_{\{0 \in D\}}-\left(1-\pi_{1}\right)(1-x / \lambda)\right)+p_{I}-p_{C} \\
& -c x\left(1-\pi_{0}\right)\left(\frac{1}{1+\ln (\lambda / x) / \lambda}\right)^{\alpha}=0
\end{aligned}
$$

leading to curves of $\psi(\cdot)$ similar to what we got in Example 2. Again several solutions are possible, so we assume that the largest stable domain $D_{1}$ is reached, thanks to a valid choice of initial conditions. Let $D_{1}^{0}$ (resp. $D_{1}^{\emptyset}$ ) denote $D_{1}$ when 0 is (resp. is not) choosing the community network. There are three possibilities depending on $\Phi_{D}(0)$ and $D_{1}$ :

- If $\Phi_{D}(0) \geq 0$ when $D=\{0\} \cup D_{1}^{0}$, it means that users 0 are satisfied with the gain in price to be in the community network, and $D=\{0\} \cup D_{1}^{0}$ is the considered user equilibrium domain;

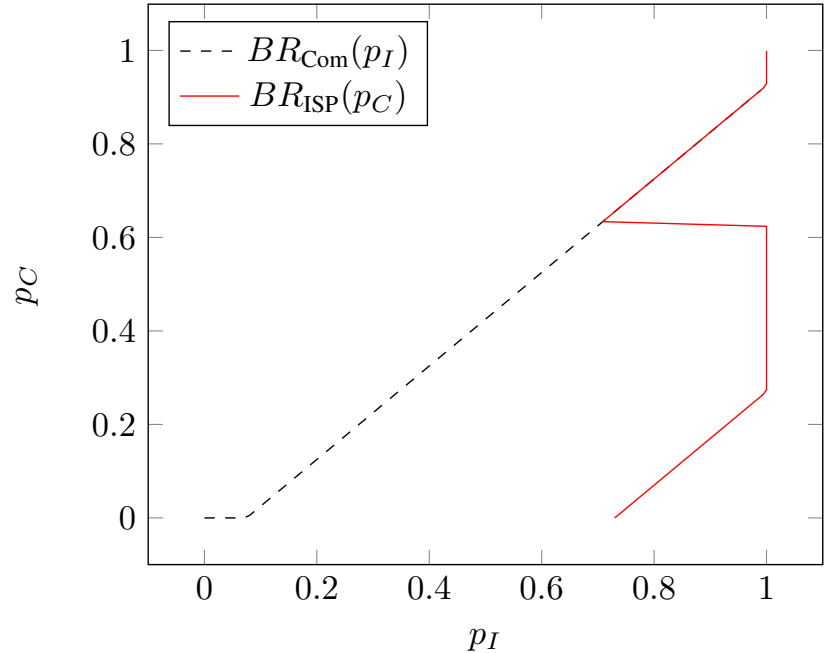

Fig. 5. Best responses in the pricing game with heterogeneous users when $\lambda=0.25, \alpha=1.2, a=1, c=1, \pi_{0}=0.7, \pi_{1}=0.1$, and $\chi_{I}=0$

- If $\Phi_{D}(0)<0$ when $D=D_{1}^{\emptyset}$, users in 0 have no interest in joining the community network and $D=D_{1}^{\emptyset}$ is an equilibrium;

- If $\Phi_{D}(0)<0$ when $D=\{0\} \cup D_{1}^{0}$ and $\Phi_{D}(0)>0$ when $D=D_{1}^{\emptyset}$, there are oscillations in the user equilibrium domain, which does not exist with the chosen strategy from the community network. In that case, we are going to consider that when computing best responses in the pricing game, operators will make use of the "worst" scenario for them in the pricing game, in terms of market share: $D=D_{1}^{\emptyset}$ for the community network, and $D=$ $\{0\} \cup D_{1}^{0}$ for the ISP.

Using the above user equilibrium domain, we can can draw the best responses of the pricing game between operators. It is for example displayed in Figure 5 for specific parameter values when the infrastructure cost for the ISP is $\chi_{I}=0$. With these numerical values, the best response of the ISP to the community network price $p_{C}$ is such that $d_{I}=1$ for $p_{C} \leq 0.63$, and $d_{I}=0.7$ (just users in 0) when $p_{C}$ is above that value. We again have a price war (each provider setting its price just below its opponent) in the high-price region. Predicting the outcome of the competition is not trivial, since following a best-response dynamics leads to a cycle (appearing in the top-right corner in the figure): prices slide downwards until reaching $\left(p_{i}, p_{C}\right) \approx(0.71,0.63)$, at which point the ISP sets $p_{I}=1$, reinitiating the cycle.

\section{REFERENCES}

[1] M. H. Afrasiabi and R. Guérin, "Exploring user-provided connectivity," IEEE/ACM Trans. on Networking, vol. 24, no. 1, 2016.

[2] M. H. Manshaei, J. Freudiger, M. Felegyhazi, P. Marbach, and J.-P. Hubaux, "On wireless social community networks," in Proc. of IEEE INFOCOM, Phoenix, AZ, USA, 2008.

[3] M. J. Osborne and A. Rubinstein, A course in game theory. MIT Press, 1994.

[4] M. H. Manshaei, P. Marbach, and J.-P. Hubaux, "Evolution and market share of wireless community networks," in Proc. of IEEE GameNets, Istanbul, Turkey, 2009. 
[5] A. Mazloumian, M. H. Manshaei, M. Felegyhazi, and J.-P. Hubaux, "Optimal pricing strategy for wireless social community networks," in Proc. of ACM NetEcon, Seattle, WA, USA, 2008.

[6] P. Maillé and B. Tuffin, Telecommunication Network Economics: From Theory to Applications. Cambridge University Press, 2014.

\section{APPENDIX}

A. All domains on equilibrium situations have the form $D_{x}$ for homogeneous and regular mobility patterns

Proof. At an equilibrium situation, the community domain $D$ must be such that

$$
\begin{array}{ll}
\forall u \in D, & \Phi_{D}(u) \geq 0 \\
\forall u \notin D, & \Phi_{D}(u) \leq 0 .
\end{array}
$$

Let $p:=p_{I}-p_{C}$. Under the assumption that $g(v \mid u)$ does not depend on $u$ and with the expressions of $q$ and $n$ we had before, we now have $\forall u \in \Omega$,

$$
\Phi_{D}(u)=a\left(\int_{D} g(v) d v-1\right)+p-c g(u) \int_{D} m(v) f(v) d v .
$$

So an equilibrium domain $D$ should be made of $u$ such that

$$
g(u)<a \frac{\int_{D} g(v) \mathrm{d} v-1}{c \int_{D} m(v) f(v) \mathrm{d} v}
$$

plus possibly some of the users for which there is equality above, but which are of measure 0 under our assumption. Hence the general form of the solution $D_{x}=\{u \in \Omega \mid g(u) \leq$ $x\}$. Using that form $D_{x}$ for candidate domains, one can write $\Phi_{D_{x}}(u)$ as a function of $x$ and $g(u)$, and an equilibrium is reached when the users wanting to subscribe to the community network (currently made of $D_{x}$ ) is exactly $D_{x}$, i.e., when $x$ is a root of the function $\Psi(x):=a\left(\int_{D_{x}} g(v) d v-1\right)+p-$ $c x \int_{D_{x}} m(v) f(v) \mathrm{d} v$. 